\title{
Why do physicians prescribe antibiotics?
}

\author{
C. Ruef
}

Published online: 9 August 2011

(C) Springer-Verlag 2011

The superficial reader would answer this question thus: "Because the patient has an infection." The more informed reader knows that a relevant proportion of antibiotics is prescribed to patients that do not have a bacterial infection and, therefore, do not benefit from such antibiotic treatment. The reasons for the overprescription of antibiotics have been investigated in numerous studies. One major reason for overprescription lies in the fact that it is clinically often difficult to determine at the outset whether the patient has a bacterial infection or not. The physician is, therefore, faced with the challenge to perform a risk assessment during which he or she weighs the benefits and disadvantages of giving or withholding antibiotics. If antibiotics are not given, patients with serious bacterial infections may face the risk of rapid progression of the infection and, potentially, a lethal outcome. Under such circumstances, it is understandable that physicians may decide to err on the safe side and to prescribe antibiotics. Another situation which may result in overprescription is the pressure and the expectation of the patient or the parents of patients to receive antibiotics for a clinical illness, which, in the eye of the patient, is disturbing and calls for antibiotic treatment.

Several other studies have shown that educational activities on the proper use or misuse of antibiotics may have a positive impact on the prescribing habits of physicians. Such studies are important, as the overuse of antibiotics remains an important driving force for the development of antibiotic resistance all over the world.

C. Ruef $(\square)$

Division of Infectious Diseases and Hospital Epidemiology,

HAL 14C, University Hospital of Zurich, 8091 Zurich,

Switzerland

e-mail: christian.ruef@usz.ch
In order to better target the content of such educational activities, it is important to understand the reasons for antibiotic prescription in the physician populations of a given country or area. The study by Velasco et al. in this issue of INFECTION provides some important insight into the factors that influence physicians' decisions to prescribe antibiotics, which are not directly related to the clinical situation of patients, but are, rather, influenced by sociobehavioral characteristics [1]. Among the parameters identified in this survey of German physicians, which are not directly related to the physician-patient relationship, male sex, practicing in the eastern states of Germany, and having had negative experiences with failed therapies were predictors for a higher likelihood of antibiotic prescription compared with colleagues that did not share these characteristics. Although the results of this survey with a response rate of $33 \%$ must be interpreted with caution, the findings are certainly interesting and point out a need to take into account such socio-behavioral factors when planning continuous medical education activities on the topic of antibiotic use.

\section{Ruef, Editor-in-Chief, INFECTION}

\section{Reference}

1. Velasco E, Espelage W, Faber M, Noll I, Ziegelmann A, Krause G, Eckmanns T. A national cross-sectional study on socio-behavioural factors that influence physicians' decisions to begin antimicrobial therapy. Infection. 2011;39. doi:10.1007/s15010011-0137-1 